\title{
THE THEORY OF ANALYTIC FUNCTIONS IN NORMED ABELIAN VECTOR RINGS
}

\author{
BY \\ EDGAR R. LORCH
}

Introduction. The problem of determining the broadest domain over which the classic results of analytic function theory are valid has attracted attention continuously since the time of Hamilton's essays in that direction with quaternions $\left({ }^{1}\right)$. The contributions to this subject converge from many directions; matrix theory, the theory of hypercomplex numbers, the theory of integral equations, and others, provide the frame for the investigations $\left({ }^{2}\right)$. It is established in the present paper that classical function theory can be extended to normed abelian vector rings. This result was known for finite dimensional rings $\left({ }^{3}\right)$. The infinite dimensional case requires for its elaboration certain basic information concerning the structure of bounded linear transformations. A surprising feature of the present development is that the methods as well as the results of the classic theory can be carried over almost without change $\left({ }^{4}\right)$.

In the preliminary section are derived the elementary properties of the rings here considered. Then the Cauchy theory is elaborated. The theory of ideals is examined insofar as it bears strongly on present questions. Use is made frequently of the notion of maximal ideal; the existence and principal properties of these were established by Gelfand $\left({ }^{5}\right)$. Illustrative of the results here obtained are Theorems 6 and 9. The exponential and logarithmic func-

Presented to the Society, February 28, 1942, and April 24, 1943; received by the editors November 23, 1942.

(1) In his Vorlesungen über die Entwicklung der Mathematik im 19. Jahrhundert (Julius Springer, Berlin, 1927), Felix Klein writes: "Das letzte Ziel (of quaternion theory) war-und ist-eine quaternionistische Funktionentheorie, von der man ganz neue, gewaltige umfassende Aufschlüsse für die ganze Mathematik erhoffte. Zur Förderung dieser zwar nicht deutlichen aber glaübig angenommenen Ziele wurde gar 1895 ein 'Weltbund zur Förderung der Quaternionen' gegründet." See vol. 1, p. 189.

(2) A large though necessarily incomplete list of references is given by J. A. Ward, $A$ theory of analytic functions in linear associative algebras, Duke Math. J. vol. 7 (1940) pp. 232-248.

(8) G. Scheffers studied functions analytic over commutative and associative algebras with a finite basis-Verallgemeinerung der Grundlagen der gewöhnlichen komplexen Funktionen, Leipziger Berichte vol. 45 (1893) pp. 838-848; vol. 46 (1894) pp. 120-134. Scheffers' results were extended by P. W. Ketchum, Analytic functions of hypercomplex variables, Trans. Amer. Math. Soc. vol. 30 (1928) pp. 641-667.

(4) A very characteristic change in the present development is that the equality for complex numbers $|a \cdot b|=|a| \cdot|b|$ is replaced by the inequality in rings $|a \cdot b| \leqq|a| \cdot|b|$. It can readily be seen that certain classic proofs of function theory (convergence of integrals and series) are not affected by this change.

(5) I. Gelfand, Normierte Ringe, Rec. Math. (Mat. Sbornik) N.S. vol. 9 (1941) pp. 1-24. 
tions are discussed. The importance of considerations of reducibility here may be judged from the fact that $\exp (z)$ is simply periodic if and only if the ring is irreducible. The problem of embedding an element in a continuous multiplicative group is treated completely in Theorem 11. Finally, separation in a ring by a rectifiable curve is discussed in the last section: separation and irreducibility are interrelated concepts. The present paper is based on an analysis of spectra of linear transformations which the author has recently published $\left({ }^{6}\right)$. At certain points theorems are stated without proof as similar results appear elsewhere in the literature. In this respect the work of Gelfand is particularly to be cited. There is interest in noting that certain of his results (for example the theory of the radical) may be obtained equally from our work by the simple device of obtaining a representation of the given abstract ring by means of a ring of transformations (Theorem 1 ).

Vector rings. A collection $\Re$ of elements $a, b, z, \cdots$ is called a normed abelian vector ring if $\left({ }^{7}\right)$ :

(a) $\Re$ is a normed linear complex vector space.

(b) $\Re$ is a commutative ring with unit $e$.

(c) The norm function defined over $\Re$ satisfies the inequality

$$
|a \cdot b| \leqq|a| \cdot|b| \text {. }
$$

Elementary properties of these rings will be derived rapidly. The inequality $|a b-a c| \leqq|a| \cdot|b-c|$ shows that multiplication is continuous in the topology defined in $\Re$ by the norm. If $a^{-1}$ represents the reciprocal (in transformation theory, the inverse) of $a$, the equation

$$
(a-z)^{-1}=a^{-1}+a^{-2} z+a^{-3} z^{2}+\cdots,
$$

valid for $|z|<\left|a^{-1}\right|^{-1}$, shows that the elements in $\Re$ possessing an inverse form an open set. It also shows that inversion is a continuous (indeed, analytic) procedure. If $a^{-1}$ exists, $a$ is called regular; in the contrary case, $a$ is called singular. The regular elements form a topological group $G$ relative to multiplication. The set $G$, being open, is the point set sum of disjoint maximal open connected sets $G_{\alpha}, G=\sum_{\alpha} G_{\alpha}\left({ }^{8}\right)$. That component containing the unit $e$ will be denoted by $G_{1}$ and will be called the principal component of $G$. If $a \in G_{1}, b \in G_{1}$, then $a b \in G_{1}$ and $a^{-1} \in G_{1}$, that is, $G_{1}$ is a group. The set $F$ of

${ }^{(6)}$ The spectrum of linear transformations, Trans. Amer. Math. Soc. vol. 52 (1942) pp 238-248. This paper will be referred to as $\mathbf{S}$. In preparing $\mathrm{S}$, the author unfortunately relied too heavily on the literature of the past decade to furnish him with a list of references. For that reason it was only after the present manuscript was submitted that he noted the brief but pointed results of F. Riesz in initiating this theory. These are to be found in his book, Les systèmes d'équations linéaires, Paris, 1913, pp. 117-121. I hope Professor Riesz will accept my sincere apologies.

${ }^{(7)}$ This definition appears in a paper by M. Nagumo, Einige analytische Untersuchungen in linearen metrischen Ringen, Jap. J. Math. vol. 13 (1936) pp. 61-80.

( ${ }^{8}$ ) An example of a ring in which the multiplicative group $G$ is not connected is the ring $\Re_{2}$ of the example discussed after Theorem 12. 
singular elements is closed. If $\Im$ is an ideal, $\Im \neq \Re$, then $\Im \subset F$. If $\Im=F, \Im$ is the radical of $\Re$ (see Theorem 6 ).

If $a$ is fixed in $\Re, z$ arbitrary, the mapping

$$
T_{a} z=a z
$$

establishes a correspondence $a \rightarrow T_{a}$ between $\Re$ and a class of bounded linear transformations defined over $\Re$; this correspondence is called the regular representation of $\Re$.

THEOREM 1. The mapping $a \rightarrow T_{a}$ of the regular representation of $\Re$ is an algebraic and topological isomorphism.

This theorem enables one to apply the theory of linear transformations to $\Re$. Thus one may say, "the complex number $\lambda$ is in the spectrum of $a$ " instead of the more cumbersome " $\lambda$ is in the spectrum of $T_{a}$ " $\left({ }^{9}\right)$.

The ring $\Re$ is reducible if $\Re$ is the disjoint sum of two rings each distinct from $\Re, \Re=\Re_{1}+\Re_{2}$. More precisely, this means that if $a \in \Re$, there exists a unique pair $a_{1} \in \Re_{1}, a_{2} \in \Re_{2}$ such that $a=a_{1}+a_{2}, a_{1} a_{2}=0$. Reducibility implies and is implied by the existence of an idempotent $j, j^{2}=j$, with $j \neq 0, j \neq e$. The rings $\Re_{1}$ and $\Re_{2}$ are given by $\Re_{1}=j \Re, \Re_{2}=(e-j) \Re$ where $e-j$ is also an idempotent. A fundamental characterization of irreducible rings is given in the following theorem.

THEOREM 2. The ring $\Re$ is irreducible if and only if the spectrum of every element in $\Re$ is a connected set.

If $\Re$ is reducible, and if $j$ is an idempotent in $\Re, j \neq e, j \neq 0$, the spectrum of $j$ consists of the two points $\lambda=0, \lambda=1$, a disconnected set. Now let $a$ be any element in $\Re$ whose spectrum is not connected. Let $C$ be a simple closed rectifiable curve in the resolvent set of $a$ such that points of the spectrum of $a$ lie both interior and exterior to $C$. The integral

$$
j=\frac{1}{2 \pi i} \int_{C} \frac{d \zeta}{\zeta e-a}
$$

evaluated over $C$ in the counterclockwise sense has the property $j^{2}=j\left({ }^{10}\right)$. By $\mathrm{S}$, Theorem $8 \mathrm{c}, \mathrm{d}$, p. $244, j \neq 0, j \neq e$, hence $\Re$ is reducible.

Clearly, if $\Re$ is finite dimensional, it is the direct sum of irreducible rings. This is not the case for infinite dimensional rings. Irreducible rings are fundamental in function theory. For example, the ring of continuous complex

(9) Throughout the paper, complex numbers will be denoted by Greek letters $\alpha, \gamma, \zeta, \lambda, \cdots$ It is worth while to compare certain expressions of abstract algebra and the corresponding expressions in transformation theory. Thus " $a$ is a null-divisor" is equivalent to " $\lambda=0$ is in the point spectrum of $a . "$

(10) See S, Theorem 6, p. 242. That the spectrum can be separated by a curve in case it is not connected is discussed under Spectral components, p. 245. 
functions defined on a finite closed real interval, or the ring of functions analytic and bounded over a region of the complex plane are irreducible $\left.{ }^{11}\right)$.

It is important to know what (complex) rings $\Re$ may be fields. The answer is provided by Theorem 3. It is also interesting to determine what rings have the property $|a \cdot b|=|a| \cdot|b|$. This is settled by Theorem 4 .

THEOREM 3. If $\Re$ is a field, it is the field of complex numbers ${ }^{12}$ ).

THEOREM 4. If in $\Re$ the norm function satisfies $|a b|=|a| \cdot|b|$ then $\Re$ is the field of complex numbers.

Using the fact that $|e|=1$, one proves that if $b \in \Re$ is regular, the spectrum of $b$ lies on the circle $|\zeta|=|b|$. Since this is also true of $b-\mu e$ where $|\mu|$ is small, the spectrum of $b$ consists of one point. Applying S, Theorems $8 \mathrm{~d}$ and 7 , it may be shown that $b$ is a scalar.

Analytic functions. Although most proofs will be omitted in this section, it will be valuable to set down salient steps in sufficient detail so that the character of the development is clearly apparent. The purpose of this is to show that not merely the results but also the methods and even the appearance of the classic theory may be transferred to $\Re$.

A variable element in $\Re$ will be denoted by $z$. A function defined over a region in $\Re$ and having values in $\Re$ will be denoted by $f(z)$. Continuity of $f(z)$ is defined in evident fashion. The topology used throughout is that of the norm, that is, the uniform topology. A function $z=z(t)$ where $z(t)$ is a continuous function defined on a real interval $0 \leqq t \leqq 1$ defines a curve. The curve is rectifiable if the sums $\sum_{t=1}^{n}\left|z\left(t_{i}\right)-z\left(t_{i-1}\right)\right|$ are bounded; here $n$ is arbitrary and $0=t_{0} \leqq t_{1} \leqq \cdots \leqq t_{n}=1$. It may be shown that if $f(z)$ is continuous in a region $H$ and if $C$ is any rectifiable curve, closed or not, in $H$, the Riemann integral $\int_{C} f(z) d z$ exists and has the usual properties.

Definition. A function $f(z)$ is said to have a derivative $f^{\prime}\left(z_{0}\right)$ at $z=z_{0}$ if for any $\epsilon>0 a \delta>0$ can be found such that for all $h$ in $\Re$ with $|h|<\delta$,

$$
\left|f\left(z_{0}+h\right)-f\left(z_{0}\right)-h f^{\prime}\left(z_{0}\right)\right| \leqq|h| \epsilon .
$$

If $f(z)$ has a derivative throughout a neighborhood of $z=z_{0}, f(z)$ is said to be analytic at $z=z_{0}$.

(11) Let $\Re$ be the ring of complex valued continuous functions $f(t), 0 \leqq t \leqq 1$, with $|f|$ $=\max |f(t)|$. The intimate connection of the concept of irreducibility with some fundamental theorems of function theory will be illustrated: the spectrum of $f(t)$ consists of the set of values assumed by $f(t)$. If $\Re$ is assumed irreducible, this is a closed connected set. If $f(t)$ assumes real values only, the set is a real closed interval. Thus $f(t)$ assumes its maximum (or minimum) on $0 \leqq t \leqq 1$. Also, $f(t)$ assumes every value between these extremes.

If $\mathfrak{Q}$ is the ring of functions $f(z)$ analytic and bounded on $|z|<1$ with $|f|=1$.u.b. $|f(z)|, \mathfrak{Q}$ is a normed abelian vector ring. Since $|z|<1$ is a connected set and $f(z)$ is continuous the spectrum of $f(z)$ is connected and $\mathfrak{Q}$ is irreducible. p. 1025.

(12) Theorems 3 and 4 have been given by S. Mazur, C.R. Acad. Sci. Paris vol. 207 (1938) 
The Cauchy theorem may be established for functions analytic in a convex region (or in a region which is the sum of a finite number of convex regions). As in the classic case it is first established for triangles. If $a$ and $b$ are in $\Re$, the line segment joining $a$ and $b$ is given by $t a+(1-t) b, 0 \leqq t \leqq 1$. The interior of the triangle determined by $a, b, c$ consists of the points $\lambda a+\mu b+\nu c, \lambda+\mu+\nu=1, \lambda>0, \mu>0, \nu>0$. The proof now proceeds in standard fashion. Incidentally, (3) is required in the form there given and not in the quotient form $\left|\Delta w / \Delta z-f^{\prime}\left(z_{0}\right)\right| \leqq \epsilon$ normally employed to define the derivative. From triangles one proceeds to arbitrary polygons; in a convex region this is trivially simple. Finally the proof is given for an arbitrary rectifiable curve.

The Cauchy formula for $f(z)$ cannot be established without specifying carefully the curve $C$ around which the integration is performed. In some sense the point $z$ must be interior to $C$. As an illustration of the phenomena which may arise, the integral (2) does not always have the expected value $e$ or 0 . However, our development needs only the fact that, for certain special curves $C$, the classic integral yields $f(z)$. If $C$ is a simple curve lying in the $z$-plane, that is, in the plane of points $z+\lambda e$ where $\lambda$ is complex and arbitrary, and if $z$ is interior to $C$ in this plane, then the Cauchy formula holds. It is valid not only for that $z$ but for all points of a neighborhood of $z$.

Finally one establishes the Taylor expansion for $f(z)$ in the usual manner. It may be seen that in this development analytic functions could be defined equivalently by (1) the uniqueness of the derivative; (2) the uniqueness of the integral (Morera's Theorem); and (3) the possibility of a power series expansion. The concept of conformality is meaningless in $\Re$ except in the case that $\Re$ is the complex number field. For conformality may be defined as follows: $\Re$ is a conformal ring if $|a z|=K_{a}|z|$ where $K_{a}$ is some constant. Considering $\Re$ a ring of transformations (or equivalently, considering that $|e|=1$ ) and setting $z=e$ yields $|a z|=|a| \cdot|z|$ for all $a, z$ in $\Re$. By Theorem 4, $\Re$ is the field of complex numbers.

A series of powers of $z-a$ (which converges for some regular $z-a$ ) is called an element. An analytic function in the large is defined as the totality of elements which are continuations of a given one. It is curious to note that one formula may define more than one analytic function. For example, if $G$ has more than one component, $1 / z$ defines an analytic function in each. One may establish Liouville's Theorem for functions analytic and bounded everywhere. From this the fundamental theorem of algebra is obtained in the following form: Given $f(z)=c_{n} z^{n}+\cdots+c_{0}, n>0, c_{n}$ regular, there exists at least one element $a$ in $\Re$ such that $f(a)$ is singular. The existence of a zero is not asserted. If $c_{n}$ is singular, $f(a)$ may be regular for all $a$.

It is seen that in this developmen't partial differen'tial equations of the Cauchy-Riemann type play no role. In the infinite dimensional case these equations would be of a most complicated type (being dependent on bases)- 
if they could be set up at all. In the finite case, the development has been carried through by means of systems of partial differential equations $\left({ }^{13}\right)$. It would seem that this circumstance contributes to a lack of transparence in the given treatment.

Ideal theory in $\Re$. In what follows, it will be at times useful, at others necessary, to employ ideal-theoretic considerations in $\Re$. The concept of maximal ideal is particularly important. A maximal ideal is one which while distinct from $\Re$ is not contained in any other ideal. Although an ideal is not necessarily a closed set, the word "ideal" will be used subsequently as an abbreviation for "closed ideal." The basic results on ideals have been given by Gelfand and can be summarized as follows $\left({ }^{14}\right)$ :

THEOREM 5. If $\Im$ is any ideal in $\Re$, the ring $\Re / \Im$ is a normed abelian vector ring. An ideal $\Omega$ can always be found which contains $\Im, \Omega \supset \Im$, and which is maximal. The ideal $\Im$ is maximal if and only if $\Re / \Im$ is the field of complex numbers.

It is of importance to note that many properties of $\Re$ remain invariant under the operation of residuation. An example will be given: if $f(z)$ is a function of $z$ analytic at $z=c$, and if $\Im$ is any ideal in $\Re$, then $\bar{f}(z)$ is a function of $\bar{z}$ in $\bar{\Re}=\Re / \Im$ analytic at $\bar{z}=\bar{c}$. For from the series

$$
f(z)=a_{0}+a_{1}(z-c)+a_{2}(z-c)^{2}+\cdots
$$

one obtains

$$
\bar{f}(z)=\bar{a}_{0}+\bar{a}_{1}(\bar{z}-\bar{c})+\bar{a}_{2}(\bar{z}-\bar{c})^{2}+\cdots .
$$

The latter series converges for some regular $\bar{z}-\bar{c}$ since $\left|\bar{a}_{n}(\bar{z}-\bar{c})^{n}\right|$ $\leqq\left|a_{n}(z-c)^{n}\right|$, hence represents an analytic function.

The following results characterize the structure of $\Re$ in terms of the spectra of the elements of $\Re$. It has been seen in Theorem 2 how the reducibility of $\Re$ can be characterized in this fashion. An element $a$ is nilpotent if for some positive integer $n, a^{n}=0$. An element $a$ is called a quasi-nilpotent if for all complex $\mu, \lim _{n \rightarrow \infty}(\mu a)^{n}=0$.

THEOREM 6. An element a is a quasi-nilpotent if and only if the spectrum of a consists of the one point $\lambda=0$. The quasi-nilpotent elements constitute an ideal $\Omega$ called the radical of $\Re$. The radical is the intersection of all maximal ideals. If $\bar{\Re}=\Re / \Re$ and $\bar{a} \in \bar{\Re}$ is the image of $a \in \Re, a$ and $\bar{a}$ have identical spectra. Thus the ring $\Re / \Omega$ is without radical.

If the spectrum of $a$ consists of the point $\lambda=0$, then by $\mathbf{S}$, Theorem 7 , $(\mu a)^{n} \rightarrow 0$ and $a$ is a quasi-nilpotent. Also if $a$ is such that $(\mu a)^{n} \rightarrow 0$ the only

(13) See footnote 3 .

(14) Gelfand, loc. cit. pp. 6, 8, 9. 
singularity of $a$ is $\lambda=0$. The remainder of the proof follows the ideas of Gelfand.

TheOREM 7. If $\Im$ is an ideal contained in the radical $\Re$ of $\Re, \Re / \Im$ is irreducible if and only if $\Re$ is irreducible.

TheOREM 8. If $f(\lambda)=\alpha_{0}+\alpha_{1} \lambda+\alpha_{2} \lambda^{2}+\cdots$ is a power series with scalar coefficients which converges absolutely for some $\lambda \neq 0$ and $a$ is any element in $\Re$ whose spectrum lies entirely within the circle in the complex plane $|\zeta|=|\lambda|$, the series $f(a)$ converges absolutely. The spectrum of $f(a)$ is the transform under $f(\lambda)$ of the spectrum of $a$.

Since the spectrum of $\lambda^{-1} a$ lies within the unit circle, by $\mathrm{S}$, Theorem 7 , $\left|\left(\lambda^{-1} a\right)^{n}\right|=\left|\left(\lambda^{-1} a\right)^{n} e\right| \rightarrow 0$. Thus for $n$ sufficiently large, $\left|a^{n}\right|<\left|\lambda^{n}\right|$ and $f(a)$ converges absolutely.

Now suppose $\lambda$ is in the spectrum of $a$. Let $\Im$ be any ideal such that $a \equiv \lambda e(\Im)$. Write $p_{n}(\lambda)=\alpha_{0}+\cdots+\alpha_{n} \lambda^{n}, n=0,1, \cdots$ Then $p_{n}(a)$ $\equiv p_{n}(\lambda) e(\Im)$. Letting $n$ increase, one has $f(a) \equiv f(\lambda) e(\Im)$ or $f(\lambda)$ is in the spectrum of $f(a)$. Next let $\mu$ be in the spectrum of $f(a)$; let $\Im$ be a maximal ideal such that $f(a) \equiv \mu e(\Im)$. If $a \equiv \lambda e(\Im)$, repetition of the previous argument yields $f(\lambda)=\mu$.

If in particular $\sum_{n-0}^{\infty}\left|\alpha_{n}\right|<\infty$ and $|a|=1$, then once more the spectrum of $f(a)$ is the transform under $f(\lambda)$ of that of $a$. An application of this result will be given.

Let $f(z)=\sum_{n=0}^{\infty} \alpha_{n} z^{n}$ be such that $\sum_{n-0}^{\infty}\left|\alpha_{n}\right|<\infty$. Then if $f(z) \neq 0$ in $|z| \leqq 1$, $1 / f(z)=\sum_{n=0}^{\infty} \beta_{n} z^{n}$ with $\sum_{n=0}^{\infty}\left|\beta_{n}\right|<\infty$.

In proof, let $\Re$ be the ring of all $f=f(z)$ for which $\sum_{n=0}^{\infty}\left|\alpha_{n}\right|<\infty$. Let $|f|=\sum_{n-0}^{\infty}\left|\alpha_{n}\right|$. Then in $\Re,|z|=1$ and clearly the spectrum of $z$ consists of all $\lambda$ with $|\lambda| \leqq 1$. Thus by the theorem, the spectrum of $f$ consists of all values assumed by $f(z)$ for $|z| \leqq 1$. Since 0 is not one of them, $1 / f(z)$ is in $\Re$.

THEOREM 9. Let $f(\lambda)=\alpha_{0}+\alpha_{1} \lambda+\alpha_{2} \lambda^{2}+\cdots(\neq 0)$ be a scalar power series convergent for all $\lambda$. Let $a \in \Re$ be such that $f(a)=0$. Then there exists a polynomial $p(\lambda)(\neq 0)$ with scalar coefficients such that $p(a)=0$. In particular if $\Re$ is irreducible and without radical, $a$ is a scalar, $a=\mu e$.

Since the spectrum of $a$ is bounded and the zeros of $f(\lambda)$ are finite in number in any bounded set, the spectrum of $a$ consists of a finite number of isolated points, $\lambda_{1}, \cdots, \lambda_{n}$. Let $j_{1}, \cdots, j_{n}$ be the idempotents

$$
j_{k}=\frac{1}{2 \pi i} \int_{c_{k}} \frac{d \zeta}{\zeta e-a}
$$

where $C_{k}$ is a circle in the complex plane centered on $\lambda_{k} e$ and sufficiently small so as to exclude the points $\lambda_{s} e, s \neq k$. Then $e=j_{1}+\cdots+j_{n}$ and $j_{k} \cdot j_{s}=0$ for $s \neq k$ (by S, Theorems $8 \mathrm{~d}$ and 5 ). 
Suppose first that $n=1$. Expand $f(\lambda)$ around $\lambda=\lambda_{1}, f(\lambda)=\beta_{0}+\beta_{1}\left(\lambda-\lambda_{1}\right)$ $+\cdots$, and $f(a)=\beta_{0} e+\beta_{1}\left(a-\lambda_{1} e\right)+\cdots$. If $\Im$ is any maximal ideal, it contains $a-\lambda_{1} e$ which is in the radical of $\Re$, hence it may be seen by a discussion modulo $\Im$ that an element $\gamma_{0} e+\gamma_{1}\left(a-\lambda_{1} e\right)+\gamma_{2}\left(a-\lambda_{1} e\right)^{2}+\cdots$ is singular if and only if $\gamma_{0}=0$. Since $f(a)=0, \beta_{0}=0$. Suppose $\beta_{1}=0, \cdots, \beta_{s-1}=0, \beta_{s} \neq 0$. Then $f(a)=\left(a-\lambda_{1} e\right)^{s}\left[\beta_{s} e+\beta_{s+1}\left(a-\lambda_{1} e\right)+\cdots\right]=0$ and $\left(a-\lambda_{1} e\right)^{s}=0$. If $\Re$ is irreducible, then certainly $n=1$; if $\Re$ is without radical, then one may take $s=1$.

Now consider $n$ unrestricted. The element $\left(a-\lambda_{k} e\right) j_{k}$ belongs to the radical of the ring $j_{k} \Re$ consisting of all elements $j_{k} a, a \in \Re$. For since $j_{k}^{2}=j_{k}$, then by $\mathbf{S}$, Theorem 7 , for large $\mu$, hence for all $\mu, \mu^{n}\left(a-\lambda_{k} e\right)^{n} j_{k}=\left[\mu(a-\lambda e) j_{k}\right]^{n} \rightarrow 0$ as $n \rightarrow \infty$; then Theorem 6 is applied. Now it may be seen that for $k=1, \cdots, n$, the ring $j_{k} \Re$ is isomorphic with the ring $\bar{\Re}_{k}=\Re /\left(e-j_{k}\right)$ where the symbol $\left(e-j_{k}\right)$ represents a principal ideal. Hence since $\bar{f}(a)=0$ in $\bar{\Re}_{k}$, the previous paragraph shows that $\left(\bar{a}-\lambda_{k} \bar{e}\right)^{s_{k}}=0$ in $\bar{\Re}_{k}$, that is, $\left(a-\lambda_{k} e\right)^{s_{k}} \equiv 0\left(e-j_{k}\right)$, $k=1, \cdots, n$. Thus, writing $p(\lambda)=\prod_{k=1}^{n}\left(\lambda-\lambda_{k}\right)^{s_{k}}, p(a)$ is in the intersection of the ideals $\left(e-j_{k}\right), k=1, \cdots, n$. Since this intersection is the zero ideal, $p(a)=0$.

This theorem shows that an analogue to the Cayley-Hamilton theorem that every matrix satisfies its characteristic equation does not exist in $\Re$. Indeed the equation $f(a)=0$ implies that $a$ is finite dimensional in the sense that the ring it generates is finite dimensional.

The exponential and logarithmic functions. The function exp $(z)$ is defined by the series $\sum_{n-0}^{\infty} z^{n} / n$ ! which converges for all $z \in \Re$. Its functional equation and the formula for the derivative are readily obtained. For all $z$, exp $(z)$ lies in the principal component $G_{1}$ of $\Re$. First, the inverse of $\exp (a)$ is $\exp (-a)$; next, by the continuity of $\exp (z)$, if $C$ is a curve joining 0 to $a$, then the map of $C$ under the transformation $z \rightarrow \exp (z)$ joins $e$ to $\exp (a)$. The relationship of the exponential to reducibility is in teresting.

THEOREM 10. The function exp (z) is simply periodic if and only if $\Re$ is irreducible. More precisely, the periods of exp (z) are exactly the elements $2 \pi i \sum_{k=1}^{n} \pm j_{k}$ where the $j_{k}$ are idempotents, distinct or not.

Direct substitution in the series for $\exp (z)$ shows that $\exp (2 \pi i j)=e$ in case $j$ is any idempotent. Thus if $\exp (z)$ is simply periodic, then $\Re$ has but one idempotent (aside from 0 ), namely $e$; hence $\Re$ is irreducible. Now suppose that for a given $a$ in $\Re$, exp $(2 \pi i a)-e=0$. By Theorem 8 , the spectrum of $a$ consists of one or more of the points $\lambda=0, \pm 1, \cdots$. If $a$ has more than one point in its spectrum, then $\Re$ is reducible (Theorem 2). Suppose now that $a$ has only one point in its spectrum; by translation one may assume that $\lambda=0$ is that point. Let $b=\exp (\pi i a)$; then $b^{2}=e$ and $(b+e) / 2$ is an idempotent. If $b \neq e, b \neq-e, \Re$ is reducible. In the contrary case, since the spectrum of $a$ consists of $\lambda=0$, by Theorem 8 the spectrum of exp ( $\pi i a)+e$ 
consists of $\lambda=2$, hence $b \neq-e$ but rather $b=e$. Consider now in turn the elements $\exp (\pi i a / 2), \exp (\pi i a / 4)$, and so on; either $\Re$ is found to be reducible or the analytic function $\exp (2 \pi i a z)$ has the value $e$ for $z=e / 2^{n}, n=0,1, \cdots$. The function is identically $e$ and its derivative $2 \pi i a$ is 0 . This shows that $\Re$ is irreducible if and only if $\exp (z)$ is simply periodic.

In proof of the last statement of the theorem, let $\lambda_{1}, \cdots, \lambda_{n}$ be the points of the spectrum of $a, \lambda_{k}$ having an integral value. Let $j_{1}, \cdots, j_{n}$ be the idempotents defined for $a$ as in Theorem 9. Then $a=a j_{1}+\cdots+a j_{n}$. Also $\exp \left(2 \pi i a j_{k}\right)=e$ and $a j_{k}-\lambda_{k} j_{k}$ is in the radical of $\bar{\Re}=j_{k} \Re, k=1, \cdots, n$. Applying the preceding paragraph, $a j_{k}=\lambda_{k} j_{k}$ giving $a=\lambda_{1} j_{1}+\cdots+\lambda_{n} j_{n}$. Except for notation, this is the statement in the theorem.

The logarithm is defined by

$$
\log z=\int_{e}^{z} \frac{d z}{z}
$$

where the path of integration joining $e$ to $z$ is arbitrary but must consist of regular elements. Thus the logarithm is defined for elements in the principal component only. It may be shown that for any $a$ in $\Re$, the equation $\log z=a$ has solutions. By what precedes and by the obvious properties of the logarithm, $\log e=2 \pi i \sum_{k=1}^{n} \pm j_{k}$ (Theorem 10).

The components of $G$ and continuous groups. The components $G_{\alpha}$ of the group $G$ of regular elements in $\Re$ have been defined earlier; the principal component $G_{1}$ is that containing $e$. The group $G_{1}$ consists precisely of the elements $a$ expressible in the form $a=\exp (b), b \in \Re$. For if $b \in \Re$, then $\exp (b) \in G_{1}$; if $a \in G_{1}$, then $\log a$ exists and $a=\exp (\log a)\left({ }^{15}\right)$. For every $b$ there is a $b^{\prime}$ such that $b^{\prime} \in G_{1}$ and $\exp (b)=\exp \left(b^{\prime}\right)$. Indeed if $n$ is an integer such that $2 \pi n>|b|$, then $b^{\prime}=2 \pi i n e+b$ satisfies these conditions. Thus the map of $G_{1}$ under the exponential function is $G_{1}$ in its entirety.

A set of elements $a^{s}$, where $s$ is an exponent ranging over the real or complex numbers or over a more general range, constitutes a continuous additive group if $a^{s} \cdot a^{t}=a^{s+t}$ and if the group operation is continuous. In many investigations it is of importance to determine whether a given element in $\Re$ may be embedded in such a group.

THEOREM 11. An element $a \in \Re$ may be embedded in a continuous additive group, $a^{s} \cdot a^{t}=a^{s+t}$, if and only if $a$ is in the principal component $G_{1}$ of $\Re$. In that case the whole ring $\Re$ serves as a range of exponents. The groups are all of the form $a^{s}=\exp (s \log a)$ where $\log a$ is any determination of the logarithm.

If the elements $a^{s}$ form a continuous group, then letting $s$ have real values, $0 \leqq s \leqq 1$, one may see that $a$ is in $G_{1}$. It is known that all such continu-

(15) This result is given by Nagumo, loc. cit. p. 66, Theorem 3. 
ous groups with real exponents are of the form $a^{s}=\exp (s \log a)\left({ }^{16}\right)$. If now $s$ is allowed to range over $\Re$ an additive group is obtained which is an extension of the given group.

This concept of a general exponent seems to be new. Thus if $a$ is a finite dimensional nonsingular matrix one may consider such expressions as $a^{a}$.

THEOREM 12. If the number of components of the topological group $G$ is greater than one, it is infinite.

Suppose that $t$ is a regular element in $\Re$ not in the principal component $G_{1}$. Then the elements $t^{n}, n=0, \pm 1, \cdots$, belong to distinct components. For suppose that $t^{n_{1}}$ and $t^{n_{2}}, n_{1} \geqq n_{2}$, belong to the same component; then $t^{n_{1}-n_{2}}=t^{m}$ is in $G_{1}$. Let $t^{m}$ be embedded in a continuous group (Theorem 11), and if $m \neq 0$, let $\left(t^{m}\right)^{1 / m}=s$. Then if $u=t s^{-1}, u^{m}=e$. The spectrum of $u$ consists at most of the $m$ th roots of unity (Theorem 8). Thus $u \in G_{1}$, hence $t=s u \in G_{1}$. This implies that $m=0$ and that $t^{n}, n=0, \pm 1, \cdots$ are in distinct components.

Examples will be given to show how the regularity of an element and also the component in which it belongs are influenced by ring extensions and contractions. If $a$ is regular in $\Re$ and the resolvent set of $a$ is connected, then $a \in G_{1}$; for $a$ and $a+n e$ with $n>|a|$ lie in the same connected component of $G$ and $a+n e \in G_{1}$. The converse proposition is false. Let $\Re_{1}$ be the ring of all complex-valued continuous functions $f(t), 0 \leqq t \leqq 1$, with $|f|=\max |f(t)|$. Let $\Re_{2}$ be the subring of $\Re_{1}$ generated by exp $(2 \pi i t)$ and exp $(-2 \pi i t)$. Let $\Re_{3}$ be the subring of $\Re_{2}$ generated by $\exp (2 \pi i t)$ and 1 . Then in $\Re_{1}, \exp (2 \pi i t)$ is regular and since it is of the form $\exp (b)$ where $b \in \Re_{1}$, it is in the principal component. In $\Re_{2}$, exp $(2 \pi i t)$ is regular but not in the principal component; for if $f(t) \in \Re_{2}, f(0)=f(1)$, hence $2 \pi i t \in \Re_{2}$. In $\Re_{3}$, exp $(2 \pi i t)$ is a singular element. For if one writes $\exp (-2 \pi i t)-\sum_{s=0}^{n} \alpha_{s} \exp (2 \pi i s t)=k(t)$ where $n$ and the $\alpha_{8}$ are arbitrary, then $|k|=\max |k(t) \exp (2 \pi i t)| \geqq \mid \int_{0}^{1} k(t)$ $\cdot \exp (2 \pi i t) d t \mid=1$. That all points $\lambda$ with $|\lambda|<1$ are in the spectrum of $\exp (2 \pi i t)$ considered as an element of $\Re_{3}$ is a consequence of the next theorem.

The preceding discussion shows that spectral singularities may at times be removed by ring extensions. If $\Re_{2} \subset \Re_{1}$ and $a \in \Re_{2}$ and if $a-\lambda e$ is singular in $\Re_{2}$ but regular in $\Re_{1}, \lambda$ is called a removable spectral singularity of $a$. In case $\lambda$ is not removable, the singularity is called a permanent spectral singularity.

THEOREM 13. All points belonging to the frontier of the spectrum of an element $a$ in $\Re$ are permanent spectral singularities of $a$.

(16) The fact that any real continuous group $a^{s},-\infty<s<\infty$, is of the form $a^{8}=\exp (s b)$ is proved by Nagumo, loc. cit. p. 72, Theorem 9. See also D. S. Nathan, One-paramcter groups of transformations in abstract vector spaces, Duke Math. J. vol. 1 (1935) pp. 518-526. 
Since the scalar series $(\zeta-\lambda)^{-1}=\sum_{n-1}^{\infty} \lambda^{n-1} \zeta^{-n}$ converges absolutely for $\left|\zeta^{-1}\right|<\left|\lambda^{-1}\right|$, then by Theorem $8, \sum_{n_{-1}}^{\infty} \lambda^{n-1} a^{-n}$ converges absolutely (to $\left.(a-\lambda e)^{-1}\right)$ for any element $a$ whose spectrum lies outside the circle $|\zeta|=|\lambda|$.

Let $\Re_{2} \subset \Re_{1}$ and let $a \in \Re_{2}$. If $a-\mu e$ is regular in $\Re_{2}$, hence also in $\Re_{1}$, the convergence or divergence of the series for $([a-\mu e]-\lambda e)^{-1}$ is settled without reference to the ring to which $a$ belongs. If $\nu$ is a frontier point of the spectrum of $a$ considered as an element of $\Re_{2}$, this series cannot converge for any $\lambda$ with $|\lambda|>|\nu-\mu|$. This implies that $a-\nu e$ is singular in $\Re_{1}$ hence $\nu$ is a permanent spectral singularity $\left({ }^{17}\right)$.

An application of Theorem 13 will be given.

Let the spectrum of $a \in \Re$ consist of real points only. Then the spectrum is invariant under ring extensions and contractions.

Separation in $\Re$ by rectifiable curves. The theory of separation in $\Re$ by means of rectifiable curves resembles strongly that in the complex plane. If $C: z=z(t), 0 \leqq t \leqq 1, z(0)=z(1)$, is a closed continuous rectifiable curve (for definition see above), $C$ is called simple if $t_{1} \neq t_{2}(\bmod 1)$ implies $\left[z\left(t_{1}\right)-z\left(t_{2}\right)\right]^{-1}$ exists. If $C$ is such a curve in $\Re$ and $\Im$ is any ideal then $\bar{C}: \bar{z}=\bar{z}(t)$ is such a curve in $\bar{\Re}=\Re / \Im$. For $\bar{C}$ is clearly simple and since the norm of $\bar{z}(t)$ in $\bar{\Re}$ does not exceed the norm of $z(t)$ in $\Re, \bar{C}$ is rectifiable. In particular, if $\Im$ is maximal, $\bar{\Re}$ is the field of complex numbers and $\bar{C}$ is a simple closed rectifiable curve in the usual sense.

If $C: z=z(t)$ is as above and $a \in \Re$ is such that $z(t)-a$ is singular for some $t$, $a$ is called $C$-singular. If $z(t)-a$ is regular for all $t, a$ is called $C$-regular. There exist $C$-regular elements. For example $a=z(0)+\lambda e$, where $|\lambda|$ is sufficiently large, is $C$-regular. Since $C$ is a compact set the set of $C$-singular elements is closed, that of $C$-regular elements is open. Consider the integral

$$
u=\frac{1}{2 \pi i} \int_{c} \frac{d z}{z-a}
$$

where $a$ is $C$-regular. Let $b$ be on $C$ and let $z^{\prime}=(z-a) /(b-a)$. This substitution leaves the integral (4) unchanged in value and replaces $C$ by a simple curve $C^{\prime}$ lying in $G_{1}$ and containing $e$. Hence $u=(2 \pi i)^{-1} \log e=\sum_{k=1}^{n} \pm j_{k}$ where the $j_{k}$ are idempotents. If $\Re$ is irreducible, $u=n e$ where $n$ is an integer (Theorem 10).

Let $\Im$ be a maximal ideal and let $z(t) \equiv(t)(\Im)$. Then under the homomorphism $\Re \rightarrow \bar{\Re}=\Re / \Im$, the integral (4) is transformed into a classical integral $\bar{u}$ in the complex plane. Choosing a proper sense along $\bar{C}: \zeta=\zeta(t)$, hence along $C, \bar{u}=0$ or $\bar{u}=1$. Since $\bar{u}=n \bar{e}, n=0$ or 1 . The $C$-regular elements

(17) This result is implied by a lemma of G. Silov, On the extension of maximal ideals, C.R. (Doklady) Acad. Sci. URSS. N.S. vol. 29 (1940) pp. 83-84. Silov proves that $a-\nu e$ is a generalized divisor of zero, hence $\nu$ is a permanent spectral singularity. An element $b$ is called a generalized divisor of zero if there exist elements $c_{n}, n=1,2, \cdots$, such that $\left|c_{n}\right|=1$ and $\left|b c_{n}\right| \rightarrow 0$ as $n \rightarrow \infty$. 
for which $u=e$ will be called $C$-interior elements. Those for which $u=0$ will be called $C$-exterior. The $C$-interior elements form an open set which need not be connected. The same is true of the $C$-exterior elements. Exterior elements exist: if $b$ is on $C, b+\lambda e$ is exterior to $C$ for large $|\lambda|$. The existence of interior elements will be discussed below. In résumé, one has the following theorem.

THEOREM 14. If $\Re$ is an irreducible ring, a simple closed rectifiable curve $C: z=z(t)$ partitions by means of the integral (4) the elements $a$ of $\Re$ into three classes:

(1) The closed set of $C$-singular elements.

(2) The open set of $C$-exterior elements a for which $u=0$.

(3) The open set of $C$-interior elements a for which $u=e$.

The existence of interior elements is related to differentiability on $C$ in the following theorem.

THEOREM 15. If $C: z=z(t)$ is a simple closed rectifiable curve in $\Re$ and if for some $t$ the derivative $z^{\prime}(t)$ exists and is a regular element, then $C$ has interior points.

Suppose $z^{\prime}(t)$ exists and is regular at $t=t_{0}$; for $t-t_{0} \neq 0$, write $(z(t)$ $\left.-z\left(t_{0}\right)\right) /\left(t-t_{0}\right)=a+h$ where $a=z^{\prime}\left(t_{0}\right)$. Suppose $(a-b)^{-1}$ exists for $b \in \mathfrak{R}$, $|b|<r$ where $r$ is some constant. Choose $\epsilon, 0<\epsilon<r$, and find $v>0$ so that when $0<\left|t-t_{0}\right|<v,|h|<\epsilon$. Choose a $\delta>0$ such that for $\left|z-z\left(t_{0}\right)\right|<\delta,\left|t-t_{0}\right| \nless v$, $(z-z(t))^{-1}$ exists. Let $\tau$ be a real number not equal to $0,|\tau a|<\delta$. Then $w(t)=z(t)-z\left(t_{0}\right)-i \tau a$ is regular for all $t$. This is clearly true for $\left|t-t_{0}\right| \nless v$. For $\left|t-t_{0}\right|<v$, write $w(t)$ in the form $w(t)=\left(t-t_{0}-i \tau\right)\left[a+\left(t-t_{0}\right)\left(t-t_{0}-i \tau\right)^{-1} h\right]$ and it is seen here also that $w(t)$ is regular.

If $\Im$ is a maximal ideal in $\Re$, and $z(t) \equiv \zeta(t)(\Im)$, it may be seen that $\zeta^{\prime}\left(t_{0}\right)=\alpha$ exists and $\alpha \neq 0$. The point $\zeta\left(t_{0}\right)+i \tau \alpha$ lies within the curve $\zeta=\zeta(t)$ either for a $\tau>0$ or a $\tau<0$. Thus the integral (4) in $\bar{\Re}=\Re / \Im$ has for this choice of $\tau$ the value $\bar{u}=1$. Hence $u=e$ and $z\left(t_{0}\right)+i \tau a$ is interior to $C$.

Barnard College, Columbia University, New York, N. Y. 